\title{
The omp2 Gene of HPS-type Bacteria Cloning and Sequence Analysis Isolates from Sichuan Province*
}

\author{
Lirui Li ${ }^{1}$, Zexiao Yang ${ }^{1}$, Yin Wang ${ }^{1 \#}$, Qiu Jin ${ }^{1}$, Xulong $\mathrm{Wu}^{1}$, Rongchang Zhang ${ }^{2}$ \\ ${ }^{1}$ Sichuan Agricultural University, Sichuan Laboratory of Animal Disease and Human Health, SLADHH, Ya'an ,China \\ ${ }^{2}$ Tibet Changdu animal health and phytosanitary supervision, TCAHPS, Changdu, China \\ Email: 704029604@qq.com, ${ }^{\# 576122816 @ q q . c o m, ~}$
}

Received 2012

\begin{abstract}
In order to compare the homology and antigen of Haemophilus parasuis (HPS)4 outer membrane protein P2(omp2), we design a test with specific primers, using PCR amplification of isolates of Haemophilus parasuis from Sichuan Province(HP Sch2010), ompP2 gene will be cloned into the pGM-T vector, and transformed into E. coli DH5 $\alpha$. Identified by PCR and sequencing and analysis, the sequencing results showed that the published 4 HPS SW124 strains omp2 gene (1077bp), compared with the amplified 1086bp purpose fragment(containing omp2 genes), is relatively stable, with the nucleotide homology level $97 \%$ and amino acid homology level of $92.5 \%$. The variable regions are mainly concentrated in the three base sequences: 40-65,110-156,180-202.
\end{abstract}

Keywords: Haemophilus parasuis, virulence genes, omp2

\section{Introduction}

Haemophilus parasuis is a principal member of the genus by the Pasteurella Division Haemophilus, considered as the main pathogens of the Glaser's disease [1]. Nowadays, HPS disease became one of the major bacterial diseases affecting the swine industry. At the same time it increase the incidence of infection in pathogenic porcine reproductive and respiratory syndrome virus infection caused by immunosuppression, result in major economic losses, being a serious threat to the healthy development of the pig industry worldwide. Because of the numerous serotypes of the disease, and different serotypes cannot provide effective cross-protection [2], it makes the effective prevention of Haemophilus parasuis disease very difficult. The study showed that Omp2 HPS outer membrane is the most abundant protein [3], and the immune electron microscopy technique showed that a panel of monoclonal antibodies directed against the exposed area of omp2 particles with the complement can be highly efficient immune [4]. Therefore, our laboratory cloned the Sichuan isolates of the HPS omp2 gene, and made a comparison and analysis of its sequence, so that we can provide a close reference of tracking for Haemophilus parasuis clinical gene mutations and developing new and efficient vaccines.

\section{Materials and Methods}

\subsection{Enzymes, Reagents and Strain}

Tap polymerase and other reagents purchased were bought in Bowande Biological,limited,the pGM-19T kit and DL5000

\footnotetext{
*This study was supported by Grants from Program for Changjiang Scholars and Innovative Research Team in University(PCSIRT)IRT 0848, and "211-Projects" Shuangzhi Plan in Sichuan Agricultural University, Li Lirui,, Zhang Rongchang and Wang Yin, all should be considered as first authors.

${ }^{\#}$ Corresponding author.
}

Maker, DNA recovery reagents, plasmid extraction kit were purchased from Dalian TaKaRa Company. Haemophilus parasuis clinical isolates Sch2010 (serotype 4) from infected pig in Sichuan.

\subsection{Primer Design and Synthesis}

According to the omp2 gene sequence of SH0165 strains of Haemophilus parasuis in GenBank (access number: CP001321.1), we used biological software Oligo 6.0 and Primer 5.0 to design omp2 full genome sequence of primer 1 , synthesized by Shanghai Shenggong Biological Engineering Co., Ltd. The primer sequences were as following: upstream: 5'- ATGGGAAGGTAATGGC -3', downstream : 5'GTACTCGCTAAAGCAG -3 '.

\subsection{Amplification of omp2 Gene by PCR}

We incubated TSB broth under $37^{\circ} \mathrm{C}$ for 24 hours to collect bacteria as a template for PCR amplification, according to the conventional cloning methods of omp2 gene.

PCR reaction system $(50 \mu \mathrm{L})$ : PrimeSTAR (HS (Premix) $25 \mu \mathrm{L}$, bacteria $2.0 \mu \mathrm{L}$, upstream and downstream primer $(20$ pmol) $1.5 \mu \mathrm{L}$, sterile ultrapure water $20 \mu \mathrm{L}$.

PCR reaction conditions: $94^{\circ} \mathrm{C}$ initial denaturation for 5 minutes; $94^{\circ} \mathrm{C}$ for $45 \mathrm{~s}, 59^{\circ} \mathrm{C}$ for 1 minute, $72^{\circ} \mathrm{C}$ for $45 \mathrm{~s}, 30$ cycles; final extension at $72^{\circ} \mathrm{C}$ for 10 minutes. Observe the results by taking $5 \mu \mathrm{L}$ amplified product, using $1 \%$ agarose gel electrophoresis.

\subsection{Cloning of the omp2 Gene}

We cut the target fragment from the agarose gel under UV light, purified DNA under the Dalian TaKaRa DNA purification kit instructions. Purified products were cloned into the pGM-T 
vector and transformed into competent cells DH5 $\alpha$. LA tablet coating contains $100 \mathrm{mg} / \mathrm{L}$ ampicillin. We cultured it under $37^{\circ} \mathrm{C}$ overnight, picked a single colony, expanded into LB containing $100 \mathrm{mg} / \mathrm{L}$ of ampicillin. A small amount of plasmid was identified by PCR and sequencing.

\subsection{Analysis of the Nucleic Acid Sequence Features}

We used the NCBI BLAST tool, DNAStar 6.0 MegAlign software to deduce amino acid sequence and analyze the homology of the sequencing results.

\subsection{Analysis of Protein Sequence Features}

We used SOSUI online software[5]、TMHMM online、SignalP 4.0 Server 、 NCBI Conserved Domains 、 NetPhos2.0 、 NetNGlyc1.0、PSIpred EMBOSS explorer to analyze the target genes' hydrophobic protein trans membrane domain, signal peptide, protein domains, phosphorylation sites, N-glycosylation sites, high-level structure and codon preference, etc.

\section{Results and Analysis}

\subsection{HPS omp2 PCR Amplification Results}

We clinical isolated the HPS omp2 gene was amplified by PCR according to section II. We got the target fragment sizing about $1086 \mathrm{bp}$. The electrophoresis results are consistent with the size of the expected products, shown in Figure 1.

\subsection{Identification of Recombinant Plasmid}

Recombinant plasmid were extracted with plasmid extraction kit and identified the plasmid PCR and electrophoresis analysis, with the pGM-T vector as a control. The results are shown in Figure 2.

\subsection{Sequence Analysis Results}

1) Analysis of the nucleic acid sequence characteristics

We put our sequencing results (Figure 3) in the NCBI Blast analysis and open reading frame analysis [6], it showed that the open reading frame (ORF) of this $\mathrm{H}$. parasuis omp2 gene consists of $1086 \mathrm{bp}$, and the GC content is $36.37 \%$. The nucleotide homology is more than $97 \%$ comparing with the online standard serum four strains SW124 omp2 gene.

2) Analysis of amino acid sequence and molecular characteristics

We derived the amino acid sequence of the target gene, according to section II by using the DNAStar software, and we analyzed the amino acid sequence homology, hydrophobic features, signal peptide, and high-level structure molecular characteristics. The results showed that: the HPS omp2 gene encode 362 amino acids, and their sequence homology with 4 SW124 of strains omp2 protein was 92.5\%. The I, R and E3 amino acid were inserted in 145, 146 and 198. Mutation site is mainly concentrated in the three base sequence 40-65, 110-156, 80-202.

After the amino acid sequence analysis, we can say that the HPS Sch2010 omp2 protein belongs to the porin superfamily members. the SOSUI hydrophobicity analysis (Figure 4) showed: the average hydrophobicity value was -0.492798 , the transmembrane region was between the 4th and the 20th amino acids. The online TMHMM software predicted protein transmembrane region was the same as the SOSUI analysis (Figure 5). The SignalP4.0 Server signal peptide analysis results (see Figure 6) showed the signal peptide was cut in the 22-23aa amino acids, and the mature protein contained 339aa. NetPhos2 0 program analysis showed that when the threshold value was 0.5 , the sequence had a total of 25 potential phosphorylation sites, including 12 serine (Ser) phosphorylation sites, 4 tyrosine (Tyr) sites and 9 threonine (Thr) sites (Figure 7). At the same time, NetNGlyc1.0 program projections indicated that there were two potential N-glycosylation glycosylation sites in the sequence.

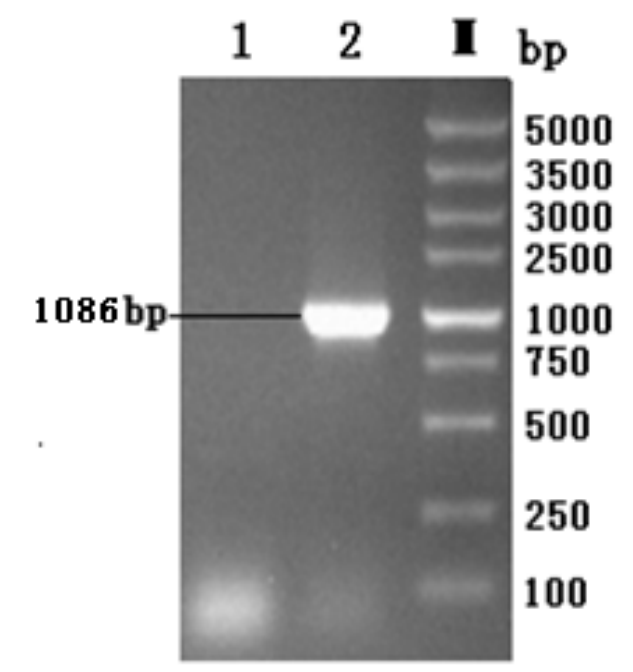

M: The DL5000 DNA maker; 1: ddH2O Negative Control; 2: HPS Sch2010

Figure1. The omp2 PCR results of HPS Sch2010

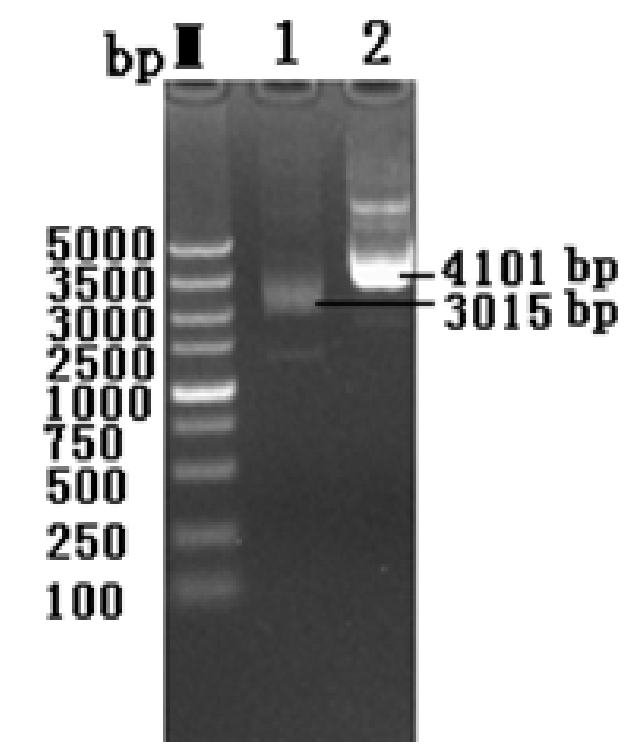

M: The DL5000 DNA maker; 1: pGM-T; 2: pGM-T-omp2 
Figure 2. The electophoresis identification results.

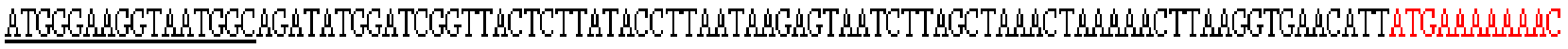

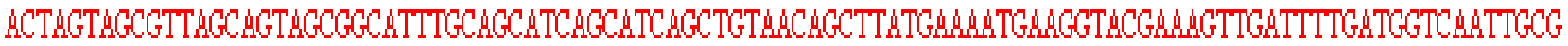

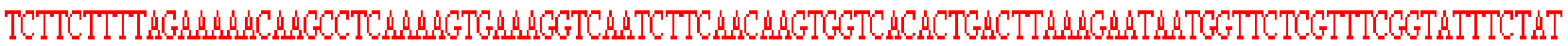

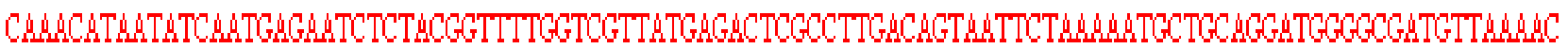
AHATMTCCTTCGTTGTTCAGGTGCTMTGGTCATGAHTTCTTTGGTAMCAGTTGTATCGTGMTAGCATTGGTCAGCTGGTTTGATA

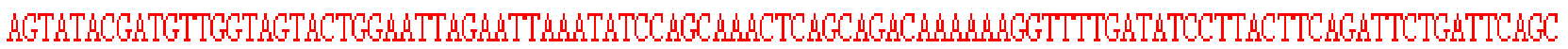

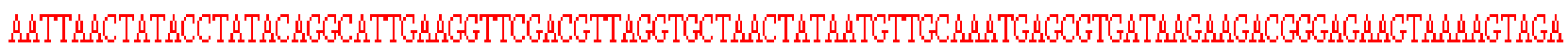
TTCTGCTAMACTGGCTTTGGTTAGGTGCTAHTACACAGCTAGATTGCGGAGTCATCTGTACTGTGCAGCAGGTTATACTCATGATGACTA

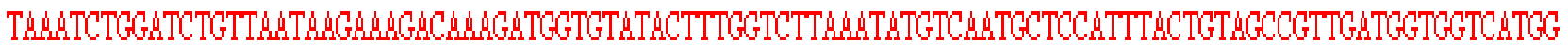

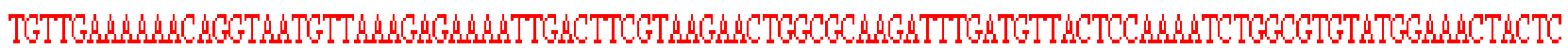

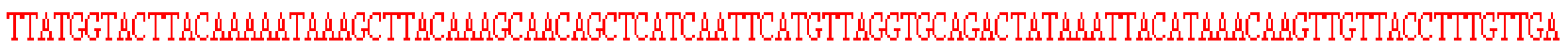

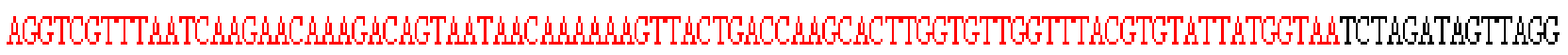

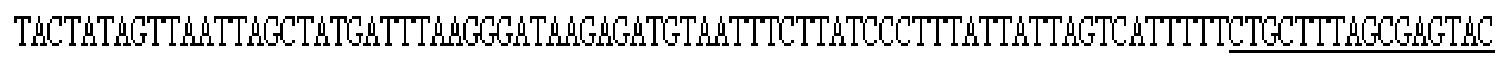

Figure 3. The underscore represents the primer position; the red font represents Sch2010 HPS omp2 target gene bands.

PSIpred procedures prediction results showed that the secondary structure composition in this HPS Sch2010 omp2 amino acid sequence, the proportion of $\alpha$-helix (Alpha-the Helix) was $28.45 \%$, $\beta$-sheet (Beta-Strand) was $29.01 \%$, $\beta$-turn was $23.76 \%$, and random coil was only $9.94 \%$.

3) Codon preference analysis

Using the CHIPS program, the HPS Sch2010 omp2 gene analysis results showed that its Nc value was 35.731, suggesting that some of its frequency of codon usage has certain differences; after CUSPS analysis, we found the gene sequence encoding the same amino acid with varying degrees of difference in codon usage frequency, such as those 4 Ala codon prefer GCA and GCT, 4 Pro codon prefer CCA, 2 Gln codon use only the CAA, Gly prefer GGT, and K prefer AAA. 15 codons in the sequence, including the TGC, TGT, GGG, ATA CTG, CCC and other appeared with zero frequency; TAA only appeared at the stop codon.

\section{Discussion}

In recent years, HPS has grown widely popular in China. It is an important pathogen of infected pigs, with numerous of different serotypes. Its cross-protective immunity is no good, which causes serious economic losses to the pig industry all over the world. Among the Haemophilus parasuis virulence factors, outer membrane protein (OMP) was the most researched one. Outer membrane protein is one of the main virulence factors of HPS[7-10] .Experimentally infected pigs with HPS produce omp antibody[11], while Tadjine M[12] prepared HPS monoclonal antibody react with 15 serotypes of HPS OMP (major outer membrane protein) and its wild-types, which indicate the outer membrane protein may have stronger immune protective effect. Meantime, Mcvicker thought that the HPS virulence strains and non-virulent strain[13] can be distinguished by omp2.

This experiment used a pair of self-designed specific primers, which were successfully amplified by PCR to be used in cloning analysis of the Sch omp2. The gene sequence analysis showed that the HPS omp2 gene fragment had the highest homology of $99 \%$, with the serum type 2 SW140 omp2 gene in GenBank (access number FJ416461.1), and their amino acid homology was $97.5 \%$; it also had the gene homology of $97 \%$ with the serum type 4 SW124 omp2 in GenBank (access number for FJ685761.1), and amino acid homology of 92.5\%. This showed that the omp2 virulence gene of Sch HPS was relatively stable but there were also certain mutations. It had higher homology with standard serotype 2 than with the standard serotype 4 . And after the secondary structure prediction and amino acid mutation site analysis we found that section 145, 146

\section{This amino acid sequence is of a $\mathbb{E E M B R A N E}$ PROTEIN which have 1 transmembrane heliz.}

\begin{tabular}{l|c|c|c|c|c|}
\hline No. & N terminal & transmembrane region C terminal & type & length \\
\hline 1 & 4 & TLWLAVASAASASSAV & 20 & PRIMARY & 17 \\
\hline
\end{tabular}


Figure 4.: HPS Sch2010 omp2 amino acid sequence Hydrophobicity analysis.

TMHMM posterior probabilities for Sequence.

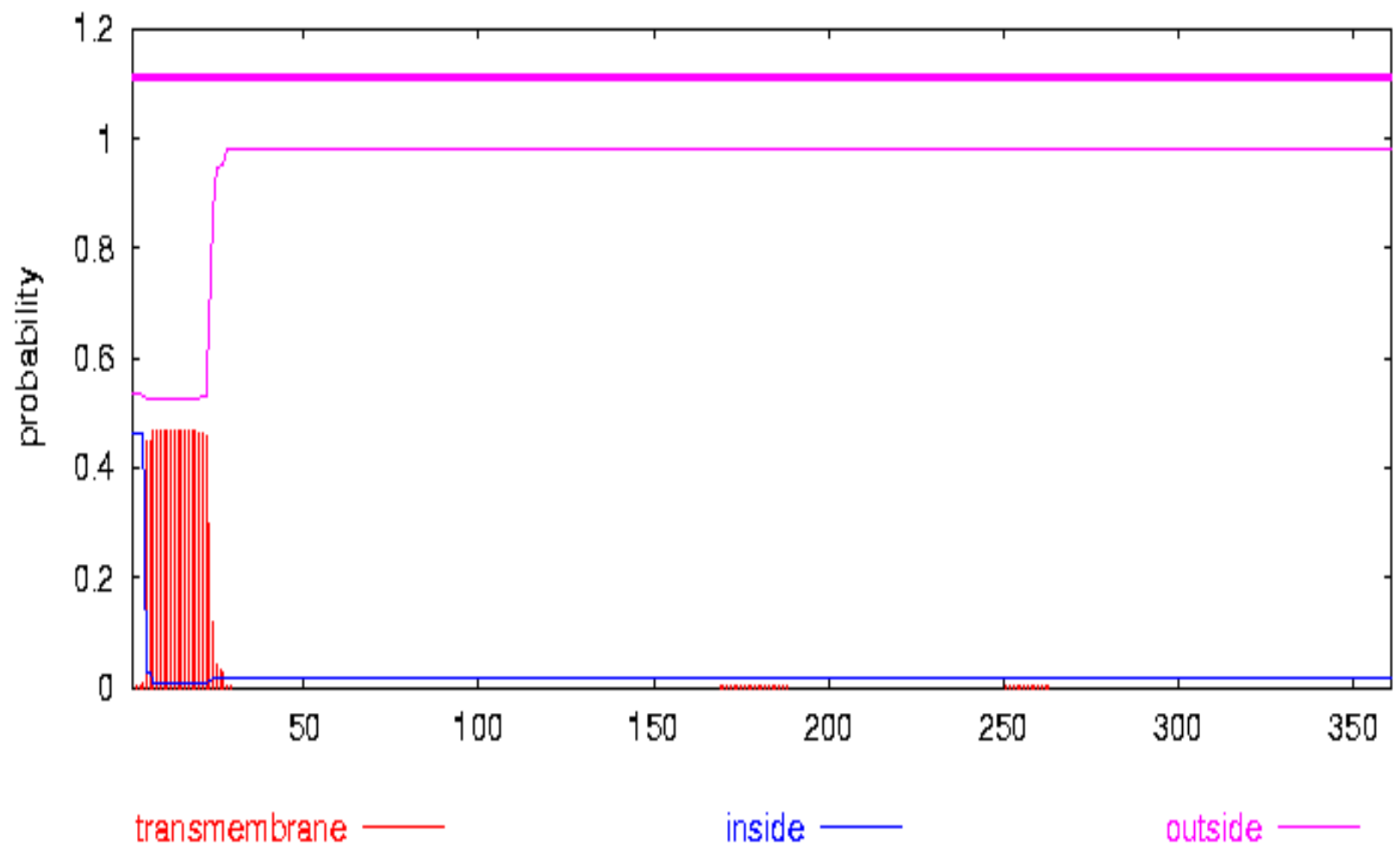

Figure 5. HPS Sch2010 omp2 amino acid sequence Protein transmembrane region predictions

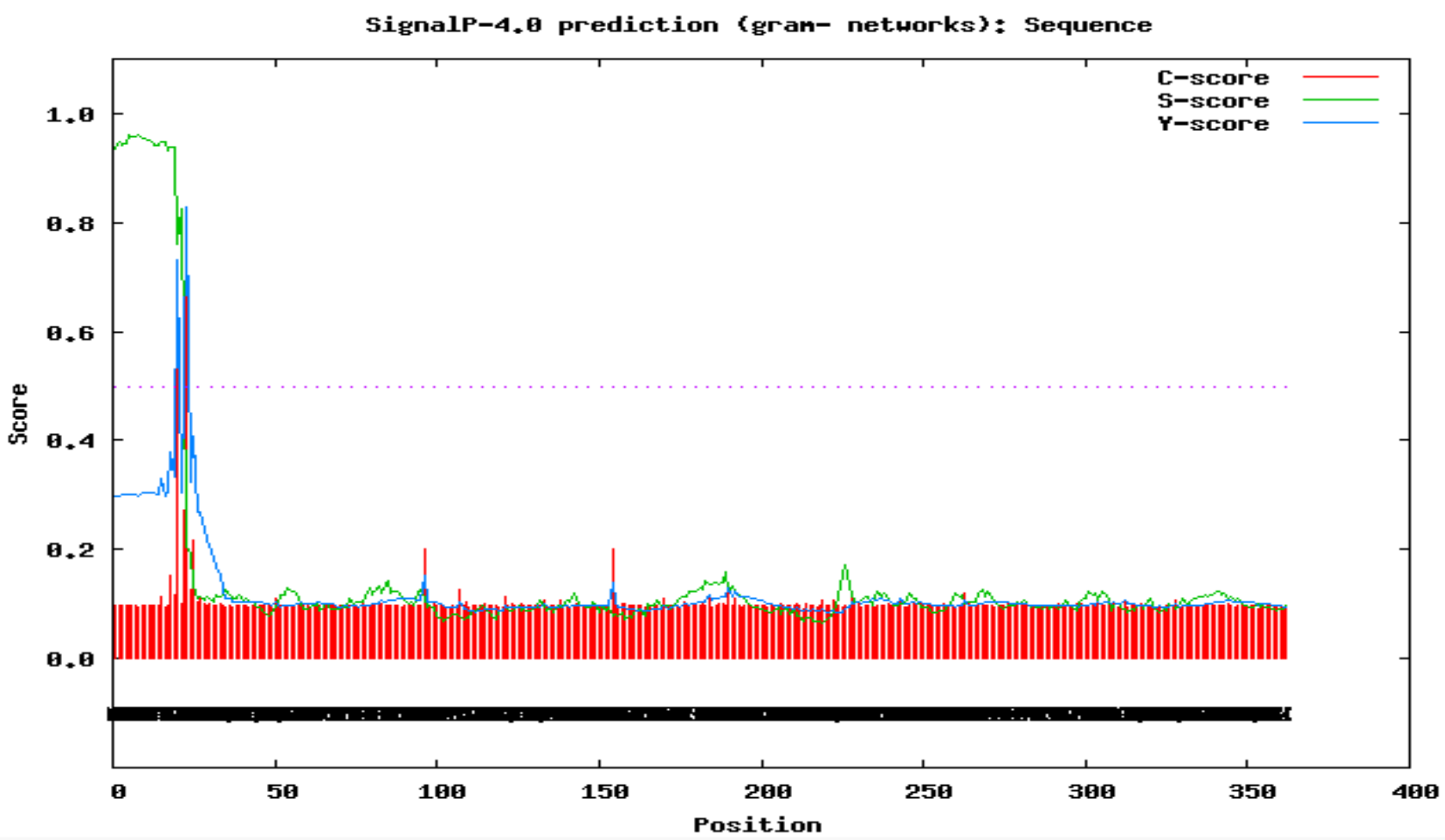


Figure 6. HPS Sch2010 omp2 amino acid sequence Signal peptide analysis

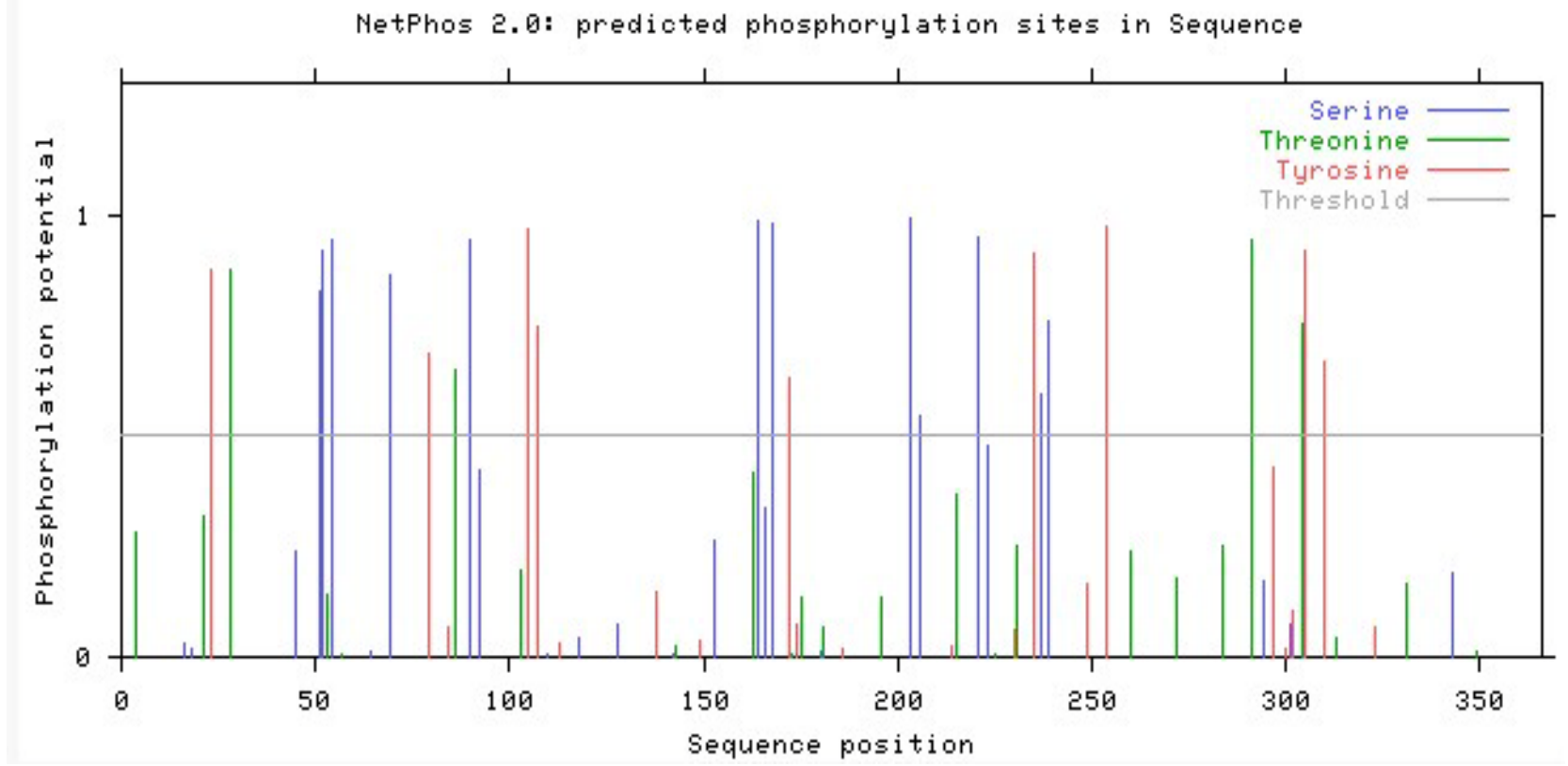

Figure 7. HPS Sch2010 omp2 amino acid sequence NetPhos2.0 program analysis

amino acid inserted just in the $\beta$-sheet area, and 198 amino acids inserted in the $\alpha$-helix, the mutation site of the focused on 40-65 and 180-202 in the $\alpha$-helix each. The isolated strain was from a large-scaled breeding farm in Suining with high fatality caused by bacteria. Whether virulence of HPS is enhanced by gene mutations, we may need further study.

Hydrophobic projections show that the peptide chains belong to hydrophilic protein and this peptide chains include transmembrane area, which indicate there may be more of a table B cells antigen in the protein. Post-translational modifications (PTMs) (e.g. phosphorylation) is a process that exists in almost all the protein formation. The modified protein often can strongly impact on the functions. This process happens primarily by the proteolysis cracking or adding one or more amino acids to a modified group so that the characteristics of the protein are changed[14,15]. This paper researched on both the 25 phosphorylation sites and 2 potential glycosylation sites existing in the amino acid sequence deduced from omp2 genes. These phosphorylation sites and potential glycosylation sites may be modified after certain PTMs process,and play important roles in the biological functions of omp2.

Meanwhile, we analyzed the codon usage preference of Sch HPS omp2 gene. And the results showed that the protein encoded the same amino acid codon preference with a large difference, which provided scientific materials and basis to the further expression of genes and related researches.

\section{REFERENCES}

[1] Simone O,Carlos P.Haemophilus parasuis:new trends on diagnosis,epidemiology and control.Vet Microbiol. vol.99, pp.75-78, 2004

[2] Oliveira A,Pina S,PerezSimo M, Marta Pérez-Simó,Simone Oliveira, Albert Bensaid.Virulence associated trimeric autotransporters of Haemophilus parasuis are antigenic proteins ex- pressed in vivo.Vet Res. vol..41,pp.26, 2010.

[3] M.Zhou,Y.Guo,J.Zhao.Identification and characterization of novel immunogenic outer membrane proteins of Haemophilus parasuis serovar 5.Vaccine.vol.27,pp.5271-5277,2009.

[4] Haase EM,Campagnari A A,Sarwar J,Shero M,Wirth M,Cumming CU,et al.Strain- specific and immunodominant surface epitopes of the P2 porin protein of nontypeable Haemophilus influenzae.Infect Immun.. vol.59,pp.1278-1284,1991.

[5] Hirokawa,Boon-Chieng,Mitaku.SOSUI classification,membrane protein two structure prediction.Bioinformatics,pp.378-379,1998

[6] Romble IT,Sykes KF,Rayner S.ORF-FINDER:a vector for high-throughput gene identification.Gene. vol.282, pp.33-41, 2002.

[7] Lillie B N,Hammerwueller J D,Macinnes J I, Mario Jacques, Anthony Hayes M.Porcine mannan-binding lectin A binds to Actinobacillus suis and Haemophilus parasuis.D Comp Immunol. vol.30,pp.954-965,2006.

[8] V.A de la Puente Redondo ,J Navas Méndez,N Garcí a del Blanco,N Ladrón Boronat,C.B Gutiérrez Martí na, E.F Rodrí guez Ferri.Typing of Haemophilus parasuis strains by PCR-RFLP analysis of the tbpA gene.Vet Microbiol. vol..92, pp.253-262, 2003.

[9] Lichtensteriger C A,Vimer E R.Purification and renaturation of membrane neuraminidase from Haemophilus parasuis.Vet Microbiol. vol..93,pp.79-87,2003.

[10] Michael A M,Karen B R,Darrell O B,Crystal L L,Tracy L N, Susan L B,et al.Characterization and comparative analysis of the genes encoding Haemophilus parasuis outer membrane proteins P2 and P5.Journal of bacteriology. vol.191,pp.5988-6002,2009.

[11] Martin de la,Fuente A J,Rodriguez-Freei E F,Martínez S,Tejerina F,Gutiérrez-Martín C B.Systemic antibody response in colostrums-deprived pigs experimentally infected with Haemophilus parasuis.Res Vet Sci. vol.86,pp.248-253,2009.

[12] Tadjine M,Mittal K R,Bourdon S,Gottschalk M.Production and characterization of murine monoclonal antibodies against Haemophilus parasuis and study of their protection role in mice.Microbiol.. vol.150,pp.3935-3945,2004. 
[13] Mcvicker J,Tabatabai L,MurielV S.Haemophilus parasuis-novel proteins-hopes for vaccine.Respiratory diseases of livestock. vol.22,pp. 2005.

[14] Nikolaj B,Thomas S P,Ramneek G, Steen G,Søren B.Prediction of post-translational glycosylation and phosphorylation of pro- teins from the amino acid sequence. Proteomics. vol.4,pp.1633-1649,2004.

[15] Mannm M,Jensen O N.Proteomic analysis of post-translational modifications.Nature Biotechnology. vol..21,pp.255-261,2003. 